\title{
Rosuvastatin prevents pressure overload-induced myocardial hypertrophy via inactivation of the Akt, ERK1/2 and GATA4 signaling pathways in rats
}

\author{
$\mathrm{XUE} \mathrm{XU}^{1}$, LEI ZHANG ${ }^{2}$ and JIANGJIU LIANG ${ }^{1}$ \\ Departments of ${ }^{1}$ Health Care and ${ }^{2}$ Orthopedics, Shandong Provincial Qianfoshan Hospital, \\ Affiliated Hospital of Shandong University, Shandong University, Jinan, Shandong 250014, P.R. China
}

Received January 28, 2013; Accepted May 29, 2013

DOI: $10.3892 / \mathrm{mmr} .2013 .1548$

\begin{abstract}
Pressure overload-induced myocardial hypertrophy is associated with a poor prognosis in humans and contributes to the development of cardiac arrhythmias, diastolic dysfunction and ultimate congestive heart failure. 3-Hydroxy-3-methylglutaryl-CoA (HMG-CoA) reductase inhibitors, also known as statins, have been previously shown to induce regression of myocardial hypertrophy in aortic banding models. However, there is limited knowledge regarding the underlying molecular mechanisms. Therefore, we hypothesized that the myocardial hypertrophy-related signaling pathways protein kinase B (Akt), extracellular signal-regulated kinases 1 or 2 (ERK1/2) and GATA binding protein 4 (GATA4) activation pathways constitute targets of rosuvastatin (RSV). Therefore, the above-mentioned activation pathways were hypothesized to be involved in the regression of pressure overload-induced myocardial hypertrophy treated by RSV. Twenty-eight Wistar rats were randomly allocated into 4 groups: the sham operation-vehicle (SH-V), abdominal aortic constriction-vehicle (AAC-V), abdominal aortic constriction-RSV $10 \mathrm{mg} / \mathrm{kg} / \mathrm{day}$ (AAC-LO) and the abdominal aortic constriction-RSV $20 \mathrm{mg} / \mathrm{kg} /$ day (AAC-HI) group. Following the establishment of the abdominal aorta constriction model, we investigated the effect of RSV, a new hydrophilic statin, on abdominal aortic constriction-induced myocardial hypertrophy as well as the underlying intercellular signaling pathways after 5 days and 4 weeks of drug intervention. Moreover, echocardiographic features and the left ventricular weight to final body weight ratio (LVW/BW) were determined. Cross-sectional areas (CSAs) of cardiomyocytes were assessed
\end{abstract}

Correspondence to: Professor Jiangjiu Liang, Department of Health Care, Shandong Provincial Qianfoshan Hospital, Affiliated Hospital of Shandong University, Shandong University, 16766 Jingshi Road, Jinan, Shandong 250014, P.R. China

E-mail: liangjiangjiu@163.com

Key words: rosuvastatin, myocardial hypertrophy, pressure overload, Akt, extracellular signal-regulated kinase 1/2, GATA binding protein 4 by hematoxylin and eosin (H\&E) staining. Atrial natriuretic factor (ANF), $\beta$-myosin heavy chain ( $\beta$-MHC) and peroxisome proliferator-activated receptor $\alpha$ (PPAR $\alpha)$ messenger RNA (mRNA) expression was assessed using RT-PCR. The phosphorylation of Akt, ERK1/2 and GATA4 were also examined using western blot analysis. Our results showed that RSV significantly attenuates pressure overload-induced myocardial hypertrophy by preventing myocardial hypertrophy-related activation of Akt, ERK1/2 and GATA4 signaling pathways.

\section{Introduction}

Chronic pressure overload induces myocardial hypertrophy, also called 'pathological' cardiac hypertrophy, which is compensatory. Excessive hypertrophy, however, is associated with significantly poor prognosis including increased cardiac morbidity, mortality and ultimate heart failure in humans $(1,2)$. 3-Hydroxy-3-methylglutaryl-CoA (HMG-CoA) reductase inhibitors, also known as statins, exert a lipid-lowering effect in the blood which has been extensively investigated (3). Additionally, recent experimental evidence indicates that certain statins restore or improve endothelial function (4), decrease smooth muscle cell (SMC) content and collagen accumulation in atherosclerotic plaques (5), play an anti-inflammatory role associated with acute coronary events (6) and activate the expression of peroxisome proliferator-activated receptors (PPARs) $(7,8)$. Moreover, according to previous studies, statins have been shown to attenuate cardiac hypertrophy (9) and to delay the progression from cardiac hypertrophy to failure under pressure overload in aortic banding models $(4,10)$. Additional evidence suggests that phosphorylated Akt/glycogen synthase kinase $3 \beta$ (GSK3 $\beta$ ), extracellular signal-regulated kinases 1 or $2($ ERK1/2) and GATA binding protein 4 (GATA4) pathways play important roles in the process of cardiac hypertrophy and ventricular remodeling induced by pressure overload (11-13). However, the molecular mechanisms of action of rosuvastatin (RSV), a hydrophilic statin which is involved in the hypertrophic response to pressure overload, remains to be fully elucidated in vivo. Therefore, we hypothesized that statins inhibit cardiac hypertrophy through the inhibition of numerous activation signaling pathways differentially associated to myocardial 
hypertrophy, such as the Akt, ERK1/2 and GATA4 signaling pathways.

The present study was divided into two major parts. We firstly investigated the transition from normal to hypertrophic myocardium and evaluated the effects of RSV on rats subjected to abdominal aortic constriction. In the second part of this study, we investigated the variation of activation pathways including Akt, ERK1/2 and GATA4 in four groups. We also explored whether RSV could reverse myocardial hypertrophy of the remodeling process following long-term treatment.

\section{Materials and methods}

Reagents. RSV was kindly provided by AstraZeneca (London, UK). Primary antibodies for total ERK1/2, AKT and their phosphorylated forms were purchased from Cell Signaling Technology, Inc. (Beverly, MA, USA). GATA4 and its phosphorylated forms were purchased from Abcam (Cambridge, MA, USA). Rabbit anti-glyceraldehyde-3-phosphate dehydrogenase (anti-GAPDH) antibody was provided by Hangzhou Goodhere Biotechnology Co., Ltd. (Hangzhou, China). Secondary horseradish peroxidase (HRP)-conjugated anti-rabbit antibody was ordered from Multisciences Biotech Co., Ltd. (Hangzhou, China). Enhanced chemiluminescence (ECL) reagent was obtained from Millipore (Billerica, MA, USA). All the reagents were used according to the manufacturer's instructions.

Animals. Male Wistar rats were supplied by the Novel Pharmaceuticals Research Center of Shandong University (Jinan, Shandong, China). The study was approved by the ethics committee of Shandong Provincial Qianfoshan Hospital, Affiliated Hospital of Shandong University, Jinan, China and the protocols were in compliance with the Guidelines for the Care and Use of Laboratory Animals published by the National Academy Press (NIH publication no. 85-23, revised 1996).

Animal experimental protocols. Twenty-eight male Wistar rats, weighing 180-220 g, were allowed free access to food and water, and were maintained on a 12-h light/dark cycle at room temperature $\left(21-23^{\circ} \mathrm{C}\right)$. When the 28 male Wistar rats weighed $300 \pm 10 \mathrm{~g}$, they were randomly allocated into 4 groups: the sham operation-vehicle ( $\mathrm{SH}-\mathrm{V}, \mathrm{n}=7)$, abdominal aortic constriction-vehicle (AAC-V, $\mathrm{n}=7$ ), abdominal aortic constriction-RSV $10 \mathrm{mg} / \mathrm{kg} /$ day (AAC-LO, n=7) and the abdominal aortic constriction-RSV $20 \mathrm{mg} / \mathrm{kg} / \mathrm{day}$ (AAC-HI, $\mathrm{n}=7$ ) group. RSV was dissolved in stroke-physiological saline solution daily; the freshly prepared RSV solution was not preserved for $>30 \mathrm{~min}$ prior to gavage. Five days prior to aortic constriction, the rats of the AAC-LO and AAC-HI groups were administered various doses of RSV, while the rats of the SH-V and AAC-V groups were administered an equal volume of vehicle by a lavage needle, until 4 weeks after surgery. Drug or vehicle were administered once daily during the entire experimental period. The rats underwent aortic constriction or sham operation 5 days after initiation of gavage and were sacrificed 4 weeks following surgery. Although higher doses of RSV (30 and $50 \mathrm{mg} / \mathrm{kg} /$ day) were also administered, male Wistar rats did not suffer any side-effects, such as intestinal obstruction of the drug, during the the experiment.
Abdominal aortic constriction model. Abdominal aortic constriction-induced pressure overload has been previously described (14). The rats were anesthetized with $10 \%$ chloral hydrate $[0.3 \mathrm{ml} / 100 \mathrm{~g}$, intraperitoneal (i.p.) injection]. The adequacy of anesthesia was measured by assessing cardiac and respiratory rates as well as pattern, lash reflex and muscle relaxation. Following asepsis, abdominal aorta was exposed through a ventral median line incision. Subsequently, ligatures were placed around both the abdominal aortas $(0.5 \mathrm{~cm}$ above renal artery bifurcation) and an obtuse 22-gauge needle (outside diameter, $0.7 \mathrm{~mm}$ ) with a $4-0$ silk suture. The needle was promptly removed following constriction. The rats of the SH-V group underwent abdominal aortic constriction without ligation.

Echocardiographic evaluation. All the rats were anesthetized with $10 \%$ chloral hydrate $(0.3 \mathrm{ml} / 100 \mathrm{~g}$, i.p.) and echocardiography was performed to evaluate left ventricular function 4 weeks following the operation (15). Left parasternal short-axis views of the left ventricle were obtained using Sonos 5500 Ultrasound Machine with a S12 Pediatric Sector Probe at $10 \mathrm{MHz}$ (Hewlett-Packard Development Company, L.P., Palo Alto, CA, USA), at the level of the papillary muscles. Dimensions of end diastolic interventricular septum (IVSd), diastolic left ventricular posterior wall (LVPWd) and left ventricular ejection fraction (LVEF) were noninvasively measured. Three consecutive cycles of measurements were performed and the results were averaged (16). Echocardiography and the measurement procedure were performed in a double-blind manner.

Body weight and cardiac characteristics. All of the rats were precisely weighed prior to echocardiography and the weight recorded was considered as the terminal body weight. The rats were perfused with stroke-physiological saline solution through the left ventricle immediately after echocardiography was performed and were then sacrificed by decapitation. The hearts of decollated rats were carefully excised and washed in double distilled water. The left ventricle including the septum was carefully clipped from the atrium and the right ventricle, and then weighed. The ratio of the left ventricular weight to body weight (LVW/BW) was described as a relative ratio.

Hematoxylin and eosin $(H \& E)$ staining. For the investigation of the myocyte cross-sectional area (CSA) of the different groups of rats, the heart tissues were stained with H\&E. The cross-sections of the left ventricle of the rats in all 4 groups were submerged in $4 \%$ paraformaldehyde solution for fixation and then embedded in wax. They were transversely cut into 4- $\mu \mathrm{m}$ slices and the cross-sections were selected adequately. The slices were dehydrated through a series of graded alcohols (100, 95 and 75\%), 15 min for each process. The slices were then stained with Mayer's hematoxylin for 10-15 min and washed under running tap water for 5-10 min. Each slice was submerged in warm water until it appeared bright purple and then counterstained in eosin solution for 2-3 min. After gently washing with water, each slice was submerged in $85 \%$ alcohol, 100\% alcohol I and II for 15 min each. Finally, all of the slices were submerged in Xylene I-Xylene II, for $15 \mathrm{~min}$ each. Photomicrographs were obtained using an Olympus 
FSX100 microscope (magnification, x30; Tokyo, Japan) under the same conditions. The surplus tissues were stored at $-80^{\circ} \mathrm{C}$ for subsequent molecular biology research.

Histopathological characteristics. Myocyte CSAs in epicardial, midwall and endocardial regions were randomly selected once for each location in the rats of all 4 groups. In each selected location, a visual field of $\geq 30-50$ cardiomyocytes was observed. Thus, $\geq 100$ cardiomyocytes were observed in all the visual fields in each sample of heart tissue. The myocyte CSA (magnification, x30) was investigated using the Image-Pro Plus software (Media Cybernetics, Carlsbad, CA, USA).

RT-PCR of cellular RNA. Total cellular RNA was extracted and purified from freezing left ventricular tissues of rats using TRIzol reagent (TransGen Biotech, Beijing, China). Primers for atrial natriuretic factor (ANF), $\beta$-myosin heavy chain $(\beta-\mathrm{MHC})$ and peroxisome proliferator-activated receptor $\alpha$ (PPAR $\alpha$ ) were designed and synthesized by Sangon Biotechnology (Shanghai, China; Table I).

Oligo(dT)-primed RNA ( $2 \mu \mathrm{g})$ was reverse transcribed to cDNA using a ReverTra Ace ${ }^{\circledR}$ qPCR RT kit (Toyobo Co., Ltd., Life Sciences Department, Osaka, Japan) in a volume system of $20 \mu \mathrm{l}$. As a template for PCR, cDNA was amplified with a Taq PCR Master mix (CWBIO, Beijing, China) using a Gene-Pro $^{\text {TM }}$ PCR Thermal Cycler. The thermocycling conditions were as follows: PCR products were predegenerated at $94^{\circ} \mathrm{C}$ for $2 \mathrm{~min}$, followed by 30 cycles of $30 \mathrm{sec}$ at $94^{\circ} \mathrm{C}, 30 \mathrm{sec}$ at different annealing temperatures $\left(60^{\circ} \mathrm{C}\right.$ for $\mathrm{ANF}, \beta-\mathrm{MHC}$ and GAPDH; $62^{\circ} \mathrm{C}$ for PPAR $\alpha$ ), then $30 \mathrm{sec}$ at $72^{\circ} \mathrm{C}$, and finally a 2 -min cycle at $72^{\circ} \mathrm{C}$. Amplified PCR products were electrophoresed on $2 \%$ agarose gels and stained with ethidium bromide; the agarose gels were then visualized with a UV illuminator (Tiangen Biotech, Inc., Beijing, China). To quantify the relative density of each DNA band, all of the gel images were analyzed using ImageJ software (NIH Image, Madison, WI, USA). GAPDH was used as an internal control. The band intensities were measured and normalized to the intensity of the respective GAPDH signal. Three independent experiments were performed in each group.

Western blot analysis. Total Akt, total ERK1/2 and their phosphorylated proteins (activation forms) were obtained using RIPA Lysis Buffer [50 mM Tris (pH 7.4), $150 \mathrm{mM}$ $\mathrm{NaCl}, 1 \% \mathrm{NP}-40,0.5 \%$ sodium deoxycholate, $0.1 \%$ SDS, sodium orthovanadate, sodium fluoride, EDTA, leupeptin] containing $1 \mathrm{mM}$ phenylmethanesulfonyl fluoride (PMSF). GATA4 and its phosphorylated protein (activation form) were extracted from the nuclei of cardiomyocytes using the Nuclear and Cytoplasmic Protein Extraction kit (Beyotime Institute of Biotechnology, Haimen, China). Protein concentrations were determined by the BCA protein assay (Beyotime Institute of Biotechnology). Equivalent amounts of protein samples $(20 \mu \mathrm{g})$ from homogenates were separated by sodium dodecyl sulfate-polyacrylamide gel electrophoresis (SDS-PAGE) at a $12 \%$ acrylamide resolving gel. Separated target proteins were transferred onto polyvinylidene difluoride membranes (Millipore). The membranes were blocked with $5 \%$ bovine serum albumin (BSA) in TBST buffer $(20 \mathrm{mM}$ Tris-HCl, pH 7.6, $150 \mathrm{mM} \mathrm{NaCl}, 0.05 \%$ Tween-20) for $2 \mathrm{~h}$ at room temperature. The membranes were then probed with primary antibodies against ERK1/2, phospho-ERK1/2 (Thr202/Tyr204), Akt, phospho-Akt (Ser473) (dilution, 1:1,000), GATA4, phospho-GATA4 (Ser105) (dilution, $1 \mu \mathrm{g} / \mathrm{ml}$ ) and GAPDH (dilution, 1:1,000) followed by incubation with the proper secondary horseradish peroxidase (HRP)-conjugated antibodies (dilution, 1:5,000). Subsequently, the membranes were washed with TBST thrice at room temperature, each for $5 \mathrm{~min}$. The reactive proteins were visualized using enhanced chemiluminescence (ECL) reagent. The intensity of the bands was analysed using ImageJ software (NIH Image) and the relative expression levels of proteins were determined using proportionality of target proteins and GAPDH.

Statistical analysis. The results are presented as the mean \pm SEM. Comparisons between groups were conducted using one-way analysis of variance (ANOVA) followed by Bonferroni's post hoc test. $\mathrm{P}<0.05$ and $\mathrm{P}<0.01$ were considered to indicate statistically significant and highly significant differences, respectively, for all the analyses. All of the statistical analyses were conducted with SPSS 17.0 statistical software package (SPSS, Inc., Chicago, IL, USA).

\section{Results}

Echocardiographic analysis. Echocardiographic analysis indicated an increased thickness of IVSd $(2.31 \pm 0.12$ vs. $1.73 \pm 0.09 \mathrm{~mm}, \mathrm{P}<0.01)$ and LVPWd $(2.15 \pm 0.07$ vs. $1.68 \pm 0.09 \mathrm{~mm}, \mathrm{P}<0.01)$ in vehicle-treated banded rats compared with rats in the sham-operated group 4 weeks following surgery. Treatment with RSV at a low and high dose resulted in decreased IVSd $(1.95 \pm 0.08$ and $1.84 \pm 0.04$ vs. $2.31 \pm 0.12 \mathrm{~mm}, \mathrm{P}<0.01)$ and LVPWd $(1.84 \pm 0.06$ and $1.77 \pm 0.04$ vs. $2.14 \pm 0.07 \mathrm{~mm}, \mathrm{P}<0.01)$ compared with rats in the untreated aortic banding group 4 weeks following surgery. Among the rats with aortic banding, LVEF evaluated by transthoracic echocardiography was found to be significantly decreased compared with the sham-operated rats $(69.41 \pm 2.85$ vs. $91.49 \pm 2.18 \%, \mathrm{P}<0.01)$. Additionally, $\mathrm{LVEF}$ of heart tissues from rats in the RSV-treated aortic banding group was higher compared with vehicle-treated banded rats $(85.07 \pm 1.87$ and $89.83 \pm 1.11$ vs. $69.41 \pm 2.85 \%, \mathrm{P}<0.01) 4$ weeks following surgery (Table II).

Animal characteristics ( $L V W / B W)$. LVW/BW was determined to evaluate the extent of myocardial hypertrophy. Constriction of abdominal aorta led to higher LVW/BW compared with the ratio of the sham-operated rats $(3.14 \pm 0.39$ vs. $1.93 \pm 0.17 \mathrm{mg} / \mathrm{g}$, $\mathrm{P}<0.01) 4$ weeks following surgery. A significantly decreased LVW/BW was observed in RSV-treated aortic banding rats when compared with the ratio of the vehicle-treated banded rats $(2.36 \pm 0.55$ vs. $3.14 \pm 0.39 \mathrm{mg} / \mathrm{g}, \mathrm{P}<0.05$ and $2.15 \pm 0.06$ vs. $3.14 \pm 0.39 \mathrm{mg} / \mathrm{g}, \mathrm{P}<0.01) 4$ weeks following surgery.

Assessment of cardiomyocyte CSA. As expected, following histological analysis, a $406 \%$ increase in myocyte CSA of the AAC-V rats was observed compared with the CSA of SH-V rats 4 weeks following surgery. The CSA of RSV-treated banded rats treated with low and high RSV doses was decreased by $\sim 39$ and $66 \%$, respectively, compared with the CSA of the AAC-V rats 4 weeks following surgery (Fig. 1). 
Table I. Primer sequences used for the target genes and internal standard GAPDH gene in rats.

\begin{tabular}{llc}
\hline Gene & \multicolumn{1}{c}{ Primer } & Size (bp) \\
\hline PPAR $\alpha$ & F: 5'-GTGGCTGCTATAATTTGCTGTG-3' & 145 \\
& R: 5'-GGAGTTTTGGGAAGAGAAAGGT-3' & 211 \\
ANF & F: 5'-AGGAGAAGATGCCGGTAGAAG-3' & \\
& R: 5'-AGAGCCCTCAGTTTGCTTTTC-3' & 256 \\
GAPDH & F: 5'-ACAGCAACAGGGTGGTGGAC-3' & 205 \\
& R: 5'-TTTGAGGGTGCAGCGAACTT-3' \\
$\beta$-MHC & F: 5'-TCCAGAAGAGAAGAACTCCATTT-3' \\
& R: 5'-ATACTCGTTGCCCACTTTGACT-3' \\
\hline GAPDH, glyceraldehyde-3-phosphate dehydrogenase; PPAR $\alpha$, peroxisome proliferator-activated receptor $\alpha$; ANF, atrial natriuretic factor; & \\
$\beta$-MHC, $\beta$-myosin heavy chain; F, forward; R, reverse.
\end{tabular}

Table II. Echocardiographic analysis of IVSd, LVPWd and LVEF, and measurements of LVW/BW in the rats of all groups.

\begin{tabular}{llcll}
\hline Group & IVSd $(\mathrm{mm})$ & LVPWd $(\mathrm{mm})$ & LVEF $(\%)$ & LVW/BW $(\mathrm{mg} / \mathrm{g})$ \\
\hline SH-V & $1.73 \pm 0.09$ & $1.68 \pm 0.09$ & $91.5 \pm 2.2$ & $1.93 \pm 0.17$ \\
AAC-V & $2.31 \pm 0.12^{\mathrm{a}}$ & $2.15 \pm 0.07^{\mathrm{a}}$ & $69.4 \pm 2.8^{\mathrm{a}}$ & $3.14 \pm 0.39^{\mathrm{a}}$ \\
AAC-LO & $1.95 \pm 0.08^{\mathrm{a}, \mathrm{c}}$ & $1.84 \pm 0.06^{\mathrm{a}, \mathrm{c}}$ & $85.1 \pm 1.9^{\mathrm{a}, \mathrm{c}}$ & $2.36 \pm 0.05^{\mathrm{a}, \mathrm{b}}$ \\
AAC-HI & $1.84 \pm 0.04^{\mathrm{c}}$ & $1,77 \pm 0.04^{\mathrm{c}}$ & $89.9 \pm 1.1^{\mathrm{c}}$ & $2.15 \pm 0.06^{\mathrm{c}}$ \\
\hline
\end{tabular}

Results are expressed as the mean \pm SEM. ${ }^{a} \mathrm{P}<0.01 \mathrm{vs}$. SH-V group; ${ }^{b} \mathrm{P}<0.05,{ }^{\mathrm{c}} \mathrm{P}<0.01 \mathrm{vs}$. AAC-V group. IVSd, diastolic interventricular septum; LVPWd, diastolic left ventricular posterior wall; LVEF, left ventricular ejection fraction; LVW/BW, left ventricular weight to body weight ratio; SH-V, sham operation-vehicle control group; AAC-V, abdominal aortic constriction-vehicle control group; AAC-LO, abdominal aortic constriction-RSV $10 \mathrm{mg} / \mathrm{kg}$ group; AAC-HI, abdominal aortic constriction-RSV $20 \mathrm{mg} / \mathrm{kg}$ group.

ANF, $\beta$-MHC and PPAR $\alpha$ mRA expression. In addition to the morphological changes, ANF and $\beta$-MHC mRNA expression levels as hypertrophic markers were significantly increased in rats with aortic banding compared with sham-operated rats $(\mathrm{ANF} / \mathrm{GAPDH}, 1.09 \pm 0.08$ vs. $0.26 \pm 0.05 ; \mathrm{P}<0.01$ and $\beta$-MHC/GAPDH, $1.19 \pm 0.07$ vs. $0.32 \pm 0.02$; $\mathrm{P}<0.01)$. However, PPAR $\alpha$ expression was decreased in aortic banded rats compared with the sham-operated rats $(\mathrm{PPAR} \alpha / \mathrm{GAPDH}$, $0.60 \pm 0.07$ vs. $1.21 \pm 0.03 ; \mathrm{P}<0.01)$. Furthermore, $\mathrm{ANF}$ and $\beta$-MHC expression levels were decreased according to the RSV doses used in banded rats compared with vehicle-treated rats $(\mathrm{ANF} / \mathrm{GAPDH}, 0.73 \pm 0.04$ and $0.53 \pm 0.03$ vs. $1.09 \pm 0.08$; $\beta-\mathrm{MHC} / \mathrm{GAPDH}, 0.63 \pm 0.04$ and $0.46 \pm 0.03$ vs. $1.19 \pm 0.07$; $\mathrm{P}<0.01$ ), in contrast with PPAR $\alpha$ mRNA expression, which was significantly increased (PPAR $\alpha / \mathrm{GAPDH}, 0.99 \pm 0.04$ and $1.02 \pm 0.04$ vs. $0.60 \pm 0.07 ; \mathrm{P}<0.01)$. No significant differences in PPAR $\alpha$ expression were observed between the two RSV-treated groups ( $\mathrm{P}>0.05$; Fig. 2).

Analysis of Akt, ERK1/2 and GATA4 protein activation. As shown in Figs. 3 and 4, quantitative total Akt and ERK1/2 protein expression (relative identities compared with GAPDH) were not significantly altered among the 4 groups (P>0.05). Phospho-Akt/Akt (p-Akt/Akt), (phospho-ERK1/2)/ ERK1/2 [(p-ERK1/2)/ERK1/2] and phospho-GATA4/GATA4 (p-GATA4/GATA4) relative protein levels were significantly increased in aortic banded rats compared with the sham-vehicle rats [p-Akt/Akt, $2.40 \pm 0.06$ vs. $0.74 \pm 0.05$; (p-ERK1/2)/ERK1/2, $0.71 \pm 0.06$ vs. $0.33 \pm 0.02$; p-GATA4/GATA4, $0.30 \pm 0.03$ vs. $0.06 \pm 0.02 ; \mathrm{P}<0.01]$. However, the relative expression of the three proteins was significantly reduced in the aortic banded rats treated with low and high doses of RSV compared with the sham-operated rats [p-Akt/Akt, $1.05 \pm 0.05$ and $0.83 \pm 0.04$ vs. $2.40 \pm 0.06$; (p-ERK1/2)/ERK1/2, $0.45 \pm 0.06$ and $0.41 \pm 0.01$ vs. $0.71 \pm 0.06$; p-GATA4/GATA4, $0.22 \pm 0.02$ and $0.13 \pm 0.02$ vs. $0.30 \pm 0.03, \mathrm{P}<0.01]$.

\section{Discussion}

It is well known that ventricular hypertrophy followed by heart failure constitute familiar outcomes of abdominal aortic constriction induced by pressure overload in rats $(17,18)$. Previous studies on this model have shown that $\beta$-adrenergic (AR) blockers and angiotensin converting enzyme inhibitors suppress myocardial hypertrophy $(19,20)$. To the best of our knowledge, this model was used for the first time in the present study to investigate the anti-myocardial hypertrophy effect of a novel HMG-CoA reductase inhibitor, RSV, and the potential underlying molecular mechanisms of action.

The beneficial effects of statins on left ventricular hypertrophy (LVH) regression in various hypertrophy models has been previously shown. Particularly, statins have been reported 
A

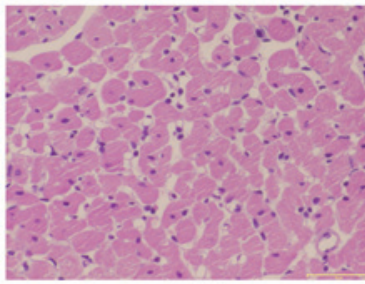

SH-V

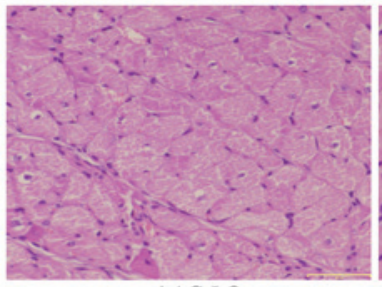

AAC-LO

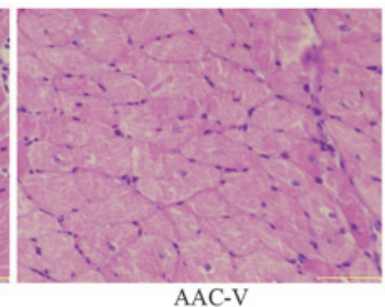

AAC-V

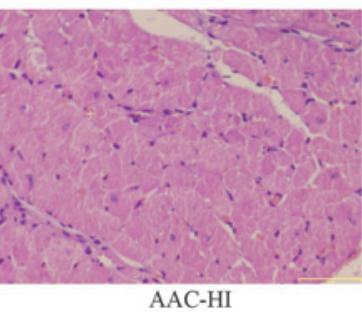

B

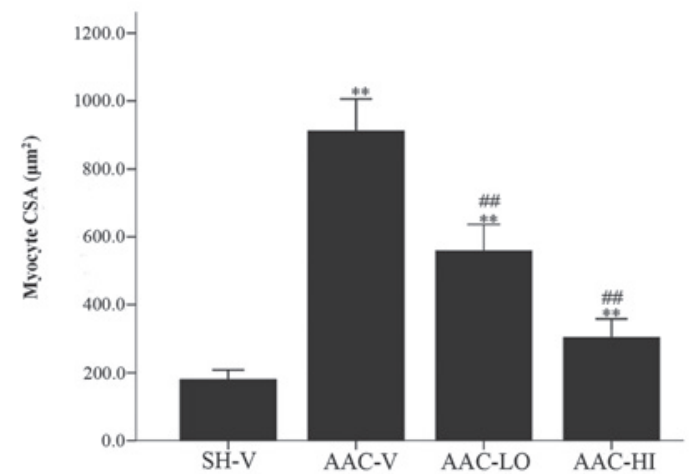

Figure 1. Mean histological CSA following H\&E staining at high magnification (x30) was assessed in the left ventricles of rats in the SH-V, AAC-V, AAC-LO and AAC-HI groups. (A) H\&E staining (magnification, $\mathrm{x} 30$ ); scale bars, $50 \mu \mathrm{m}$ and (B) quantitative analysis of mean CSA ( $\mu \mathrm{m}^{2}$ ) of cardiac myocytes in the 4 groups. Results are expressed as the mean \pm SEM. ${ }^{* *} \mathrm{P}<0.01$ vs. SH-V group; ${ }^{\#} \mathrm{P}<0.01$ vs. AAC-V group. CSA, cross-sectional area; H\&E, hematoxylin and eosin; RSV, rosuvastatin; SH-V, sham operation-vehicle control group; AAC-V, abdominal aortic constriction-vehicle control group; AAC-LO, abdominal aortic constriction-RSV $10 \mathrm{mg} / \mathrm{kg}$ group; AAC-HI, abdominal aortic constriction-RSV $20 \mathrm{mg} / \mathrm{kg}$ group.

A
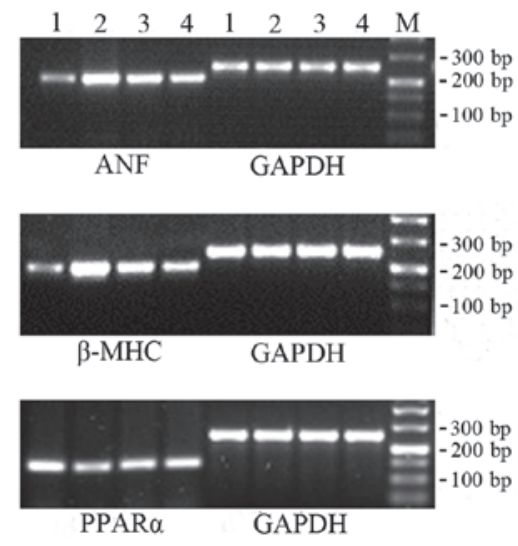

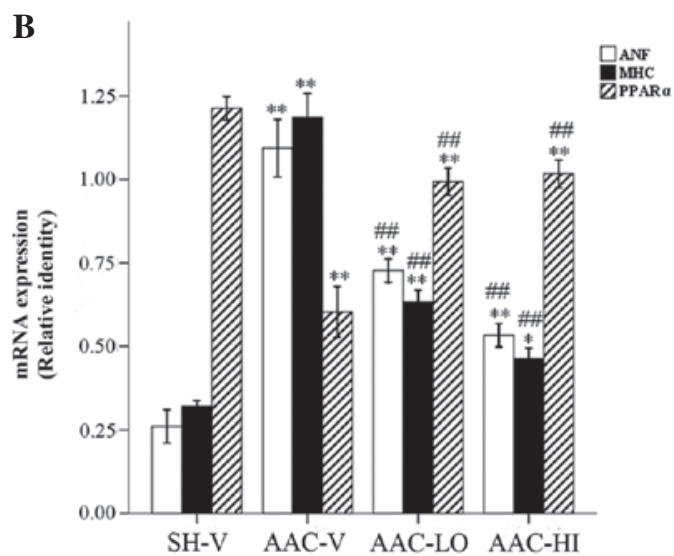

Figure 2. RT-PCR detection of ANF, $\beta$-MHC and PPAR $\alpha$ mRNA relative levels in SH-V, AAC-V, AAC-LO and AAC-HI groups. (A) mRNA expression of ANF, $\beta$-MHC and PPAR $\alpha$. M, marker; lane 1, SH-V; lane 2, AAC-V; lane 3, AAC-LO; lane 4, AAC-HI. (B) Relative qualitative identities of mRNAs were normalized to the intensity of GAPDH. The adjusted mRNA level for every group was the average of three measurements. Results are expressed as the mean \pm SEM. ${ }^{* *} \mathrm{P}<0.01$ vs. SH-V group; ${ }^{\# \#} \mathrm{P}<0.01$ vs. AAC-V group. RSV, rosuvastatin; ANF, atrial natriuretic factor; $\beta$-MHC, $\beta$-myosin heavy chain; PPAR $\alpha$, proliferator-activated receptor $\alpha$; SH-V, sham operation-vehicle control group; AAC-V, abdominal aortic constriction-vehicle control group; AAC-LO, abdominal aortic constriction-RSV $10 \mathrm{mg} / \mathrm{kg}$ group; AAC-HI, abdominal aortic constriction-RSV $20 \mathrm{mg} / \mathrm{kg}$ group.

to enhance endothelial nitric oxide synthase (eNOS) expression and to inhibit NADPH oxidase activity, which has been associated with myocardial hypertrophy endothelial function and the development of myocardial hypertrophy $(9,21)$. Statins have also been reported to inhibit myocardial hypertrophy via the ERK1/2 activation signaling pathway in spontaneously hypertensive rats (22). However, there have been controversial results regarding the effect of RSV on cardiac hypertrophy, which have been associated with the different types of animal hypertrophy models and the different experimental protocols used. RSV has been shown to inhibit cardiac hypertrophy via suppression of $\mathrm{Gh}$ and cardiac oxidative stress $(23,24)$. However, Chang et al (25) showed that RSV treatment does not reverse hypertension-induced LVH; no beneficial effects on heart failure and survival were also observed (25). The underlying mechanisms of action of RSV on abdominal aortic constriction-induced myocardial hypertrophy have yet to be fully elucidated. Therefore, the Akt, ERK1/2 and GATA4 signaling pathways were investigated in the present study.

Akt is a serine/threonine protein kinase and acts as an important pathway which is involved in the regulation of heart development. Short-term Akt activation, occurring in postnatal cardiac development and trained athletes, promotes physiological hypertrophy, while long-term activation of Akt occurring in unbounded hypertension, myocardial infarction and aortic stenosis, results in pathological hypertrophy and heart failure (26). In the present study, we found that the increased phosphorylated Akt protein level is associated with cardiac hypertrophy (pathological hypertrophy) in response to pressure overload (11). Mitogen-activated protein kinases (MAPKs) are 3-tiered kinase cascades that are classified into 3 distinct subfamilies, including extracellular signal receptor-regulated kinases (ERKs), c-jun NH2-terminal kinases (JNKs) and p38 MAPK. The three MAPK pathways 
A

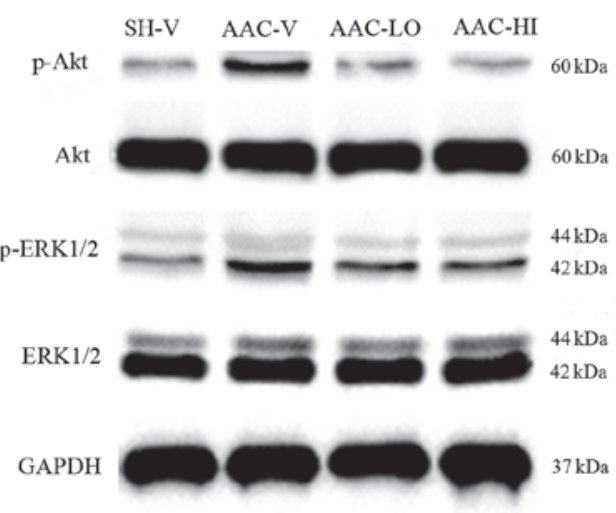

C

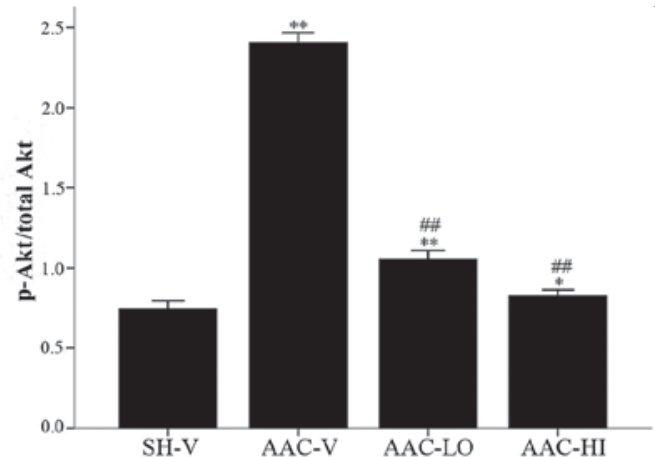

B

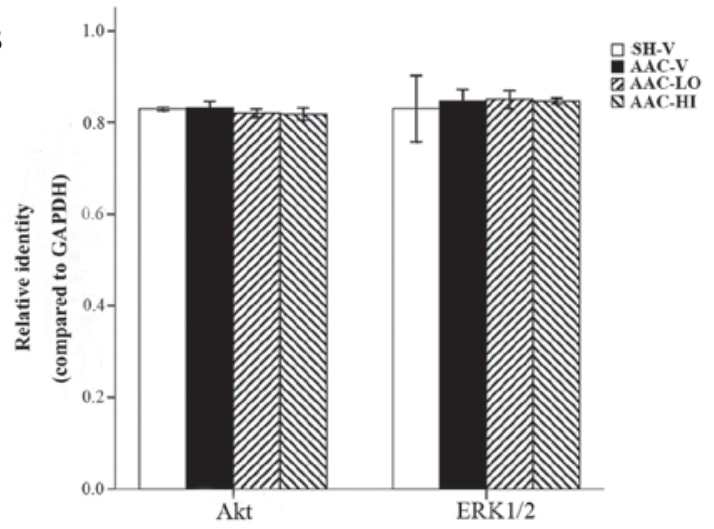

D

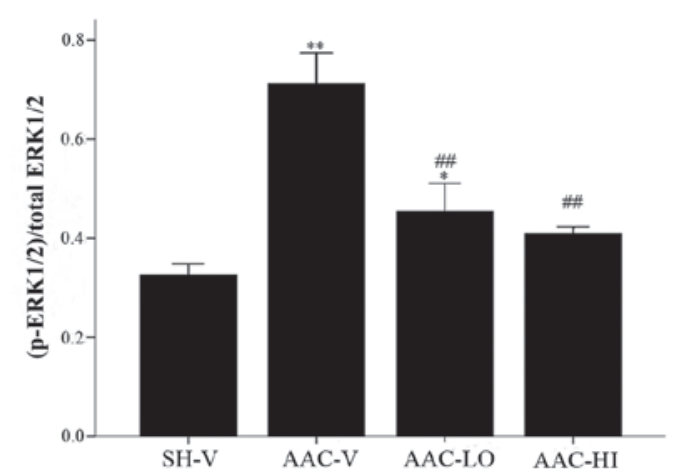

Figure 3. Western blot analysis of total Akt and ERK1/2, and their phosphorylated isoform protein levels in the cardiac tissue of rats in the SH-V, AAC-V, AAC-LO and AAC-HI groups. (A) Akt, ERK1/2, p-Akt and p-ERK1/2 protein levels. (B) Quantification of Akt and ERK1/2 compared with GAPDH. Quantification of (C) p-Akt and (D) p-ERK1/2 protein levels compared with their nonphosphorylated forms. Results are expressed as the mean \pm SEM. ${ }^{*} \mathrm{P}<0.05,{ }^{* *} \mathrm{P}<0.01$ vs. SH-V group; ${ }^{\# \#} \mathrm{P}<0.01$ vs. AAC-V group. RSV, rosuvastatin; ERK1/2, extracellular signal-regulated kinases 1 or 2 ; SH-V, sham operation-vehicle control group; AAC-V, abdominal aortic constriction-vehicle control group; AAC-LO, abdominal aortic constriction-RSV $10 \mathrm{mg} / \mathrm{kg}$ group; AAC-HI, abdominal aortic constriction-RSV $20 \mathrm{mg} / \mathrm{kg}$ group.

A

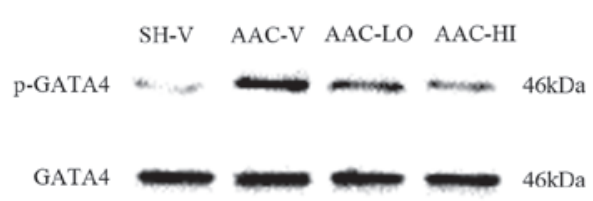

B

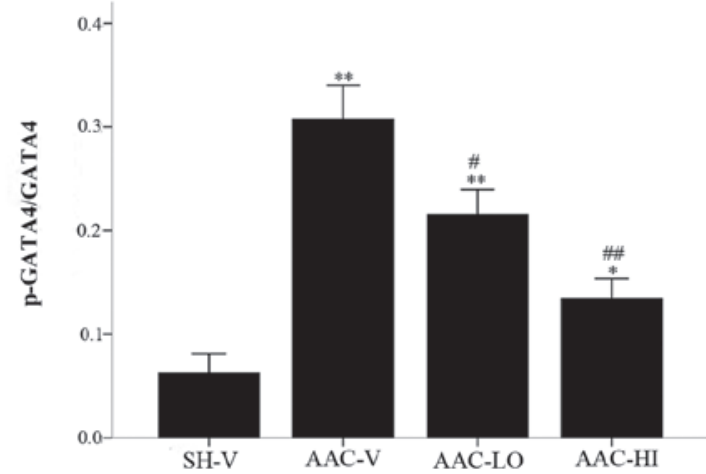

Figure 4. Nonphosphorylated and phosphorylated isoform protein levels of GATA4 in the SH-V, AAC-V, AAC-LO and AAC-HI groups. (A) GATA4 and p-GATA4 protein levels. (B) Quantification of p-GATA4 protein levels compared with GATA4. Results are expressed as the mean \pm SEM. "P<0.05, ${ }^{* *} \mathrm{P}<0.01$ vs. SH-V group; ${ }^{\# P}<0.05,{ }^{\# \#} \mathrm{P}<0.01$ vs. AAC-V group. RSV, rosuvastatin; GATA4, GATA binding protein 4; SH-V, sham operation-vehicle control group; AAC-V, abdominal aortic constriction-vehicle control group; AAC-LO, abdominal aortic constriction-RSV $10 \mathrm{mg} / \mathrm{kg}$ group; AAC-HI, abdominal aortic constriction-RSV $20 \mathrm{mg} / \mathrm{kg}$ group.

have been shown to be activated in the myocardium of mice following transverse aortic constriction (TAC) operation (12). The phosphorylated transcription factor GATA4 is required for pressure overload-induced cardiac hypertrophy in vivo (13). It has been proved that the phosphorylation of GATA4 at Ser105 directly activated by ERK1/2, improves the transcriptional activity and DNA-binding affinity of GATA4 in vivo and in vitro $(27,28)$. The activated Akt pathway managed to enforce its hypertrophic effect via GSK3 $\beta /$ GATA4 phosphorylation activity in cardiac myocytes in vivo and in vitro. Activity of the Akt signal pathway is not involved with activity of the ERK1/2 signal pathway. They are two different signal pathways which lead to GATA4 activation $(29,30)$. These data suggest that the activation of the Akt, ERK1/2 and GATA4 signaling pathways is involved in pressure overload-induced cardiac hypertrophy. Thus, we further aimed to investigate the activation of Akt, ERK1/2 and GATA4 activation signaling pathways in rats disposed by abdominal aortic constriction, and to examine 
whether RSV reduces pressure overload-induced cardiac hypertrophy by preventing the activation of these molecular pathways. PPAR $\alpha$ is a member of the nuclear receptor of ligand-activated transcription factors. Relevant studies have shown that deficiency of PPAR $\alpha$ leads to a more significant hypertrophic growth response, suggesting that PPAR $\alpha$ attenuates pathological cardiac remodeling induced by pressure overload. Thus, PPAR $\alpha$ has been suggested to exert beneficial effects on cardiac hypertrophy (31).

In the present study, abdominal aortic constriction-induced pressure overload resulted in left ventricular myocardial hypertrophy due to the increase in LVW/BW, echocardiography characteristics, cardiomyocyte area and mRNA expression levels of the hypertrophy markers ANF and $\beta$-MHC. In pressure overload-induced hypertrophic hearts, phosphorylated activation of Akt, ERK1/2 and GATA4 proteins was significantly increased, which was in agreement with the results of previous studies $(11,32,33)$. Briefly, these results showed that the phosphorylation levels of Akt, ERK1/2 and GATA4 proteins are involved in myocardial hypertrophy induced by abdominal aortic constriction. Treatment with RSV at doses of 10 and $20 \mathrm{mg} / \mathrm{kg} / \mathrm{day}$ was shown to downregulate the phosphorylation levels of Akt, ERK1/2 and GATA4 proteins, and to upregulate PPAR $\alpha$ mRNA expression in rat myocardial cells. Consequently, these may constitute the molecular mechanisms of regression to myocardial hypertrophy. Based on this observation, the effect of RSV on promoting cardiac function is suggested to be parallel with the effect of myocardial hypertrophy regression. RSV is suggested to reverse the development of cardiac hypertrophy by affecting the phosphorylation of Akt, ERK1/2 and GATA4 molecular activation signaling pathways in cardiomyocytes.

In conclusion, based on the significant decreases in left ventricular mass and relative cardiomyocyte area in the RSV-treated banded rats, RSV was shown to have cardiac anti-hypertrophic effects and to be involved in the maintenance of hemodynamic stability. Therefore, RSV suppresses myocardial hypertrophy induced by pressure overload. The underlying molecular mechanisms of action may be associated to the regulation of activation of Akt, ERK1/2 and GATA4 pro-hypertrophic signaling pathways. However, further studies are needed for the investigation of the association between Akt, ERK1/2 and GATA4 molecular activation signaling pathways. RSV treatment also increases the mRNA expression levels of PPAR $\alpha$, which is beneficial to the regression of cardiac hypertrophy. To the best of our knowledge, this is the first time that RSV has been shown to prevent and reverse cardiovascular remodelling induced by abdominal aortic constriction initiated by pressure overload. The results of the present study provide additional evidence regarding the pleiotropic effects of statins. RSV is suggested to constitute a novel drug suitable for the clinical reversal of cardiac hypertrophy. However, further studies are needed for the in-depth investigation of the role of RSV in myocardial hypertrophy.

\section{Acknowledgements}

This study was supported by the General Programs of the Natural Science Foundation of Shandong Province of China (no. ZR2010HM116).

\section{References}

1. Levy D, Garrison RJ, Savage DD, Kannel WB and Castelli WP: Prognostic implications of echocardiographically determined left ventricular mass in the Framingham Heart Study. N Engl J Med 322: 1561-1566, 1990.

2. Okin PM, Devereux RB, Jern S, et al: Regression of electrocardiographic left ventricular hypertrophy during antihypertensive treatment and the prediction of major cardiovascular events. JAMA 292: 2343-2349, 2004.

3. Auer J, Berent R, Weber T and Eber B: Clinical significance of pleiotropic effects of statins: lipid reduction and beyond. Curr Med Chem 9: 1831-1850, 2002.

4. Laufs U, Fata VL and Liao JK: Inhibition of 3-hydroxy-3-methylglutaryl (HMG)-CoA reductase blocks hypoxia-mediated down-regulation of endothelial nitric oxide synthase. J Biol Chem 272: 31725-31729, 1997.

5. Fukumoto Y,Libby P, Rabkin E, et al: Statins alter smooth muscle cell accumulation and collagen content in established atheroma of watanabe heritable hyperlipidemic rabbits. Circulation 103: 993-999, 2001.

6. Ridker PM, Rifai N, Clearfield M, et al: Measurement of C-reactive protein for the targeting of statin therapy in the primary prevention of acute coronary events. N Engl J Med 344: 1959-1965, 2001.

7. Planavila A, Laguna JC and Vázquez-Carrera M: Atorvastatin improves peroxisome proliferator-activated receptor signaling in cardiac hypertrophy by preventing nuclear factor-kappa B activation. Biochim Biophys Acta 1687: 76-83, 2005.

8. Young ME, Laws FA, Goodwin GW and Taegtmeyer H: Reactivation of peroxisome proliferator-activated receptor alpha is associated with contractile dysfunction in hypertrophied rat heart. J Biol Chem 276: 44390-44395, 2001.

9. Takemoto M, Node K, Nakagami H, et al: Statins as antioxidant therapy for preventing cardiac myocyte hypertrophy. J Clin Invest 108: 1429-1437, 2001.

10. Kameda Y, Hasegawa H, Kubota A, et al: Effects of pitavastatin on pressure overload-induced heart failure in mice. Circ J 76: 1159-1168, 2012.

11. Xu R, Lin F, Zhang S, Chen X, Hu S and Zheng Z: Signal pathways involved in reverse remodeling of the hypertrophic rat heart after pressure unloading. Int J Cardiol 143: 414-423, 2010.

12. Esposito G, Prasad SV, Rapacciuolo A, Mao L, Koch WJ and Rockman HA: Cardiac overexpression of a G(q) inhibitor blocks induction of extracellular signal-regulated kinase and c-Jun $\mathrm{NH}(2)$-terminal kinase kinase activity in in vivo pressure overload. Circulation 103: 1453-1458, 2001.

13. van Berlo JH, Elrod JW, Aronow BJ, Pu WT and Molkentin JD: Serine 105 phosphorylation of transcription factor GATA4 is necessary for stress-induced cardiac hypertrophy in vivo. Proc Natl Acad Sci USA 108: 12331-12336, 2011.

14. Siri FM: Chronic norepinephrine infusion and adrenergic function of hypertrophied hearts. Am J Physiol 248 (4 Pt 2): H485-H492, 1985.

15. Teichholz LE, Kreulen T, Herman MV and Gorlin R: Problems in echocardiographic volume determinations: echocardiographic-angiographic correlations in the presence of absence of asynergy. Am J Cardiol 37: 7-11, 1976.

16. Leskinen H, Rauma-Pinola T, Szokodi I, et al: Adaptive or maladaptive response to adenoviral adrenomedullin gene transfer is context-dependent in the heart. J Gene Med 10: 867-877, 2008.

17. Woodiwiss AJ, Tsotetsi OJ, Sprott S, et al: Reduction in myocardial collagen cross-linking parallels left ventricular dilatation in rat models of systolic chamber dysfunction. Circulation 103: 155-160, 2001

18. Hong Y, Hui SC, Chan TY and Hou JY: Effect of berberine on regression of pressure-overload induced cardiac hypertrophy in rats. Am J Chin Med 30: 589-599, 2002.

19. Ni L, Zhou C, Duan Q, et al: $\beta$-AR blockers suppresses ER stress in cardiac hypertrophy and heart failure. PLoS One 6: e27294, 2011.

20. Li J, Li P, Feng X, et al: Effects of losartan on pressure overload-induced cardiac gene expression profiling in rats. Clin Exp Pharmacol Physiol 30: 827-832, 2003.

21. Zhou MS, Jaimes EA and Raij L: Atorvastatin prevents end-organ injury in salt-sensitive hypertension: role of eNOS and oxidant stress. Hypertension 44: 186-190, 2004. 
22. Takayama N, Kai H, Kudo H, et al: Simvastatin prevents large blood pressure variability induced aggravation of cardiac hypertrophy in hypertensive rats by inhibiting RhoA/Ras-ERK pathways. Hypertens Res 34: 341-347, 2011.

23. Habibi J, Whaley-Connell A, Qazi MA, et al: Rosuvastatin, a 3-hydroxy-3-methylglutaryl coenzyme a reductase inhibitor, decreases cardiac oxidative stress and remodeling in Ren2 transgenic rats. Endocrinology 148: 2181-2188, 2007.

24. Choi EY, Chang W, Lim S, et al: Rosuvastatin inhibits norepinephrine-induced cardiac hypertrophy via suppression of Gh. Eur J Pharmacol 627: 56-62, 2010.

25. Chang SA, Kim YJ, Lee HW, et al: Effect of rosuvastatin on cardiac remodeling, function, and progression to heart failure in hypertensive heart with established left ventricular hypertrophy. Hypertension 54: 591-597, 2009.

26. Chaanine AH and Hajjar RJ: AKT signalling in the failing heart. Eur J Heart Fail 13: 825-829, 2011.

27. Liang Q, Wiese RJ, Bueno OF, Dai YS, Markham BE and Molkentin JD: The transcription factor GATA4 is activated by extracellular signal-regulated kinase 1- and 2-mediated phosphorylation of serine 105 in cardiomyocytes. Mol Cell Biol 21: 7460-7469, 2001.
28. Tenhunen O, Sármán B, Kerkelä R, et al: Mitogen-activated protein kinases $\mathrm{p} 38$ and ERK $1 / 2$ mediate the wall stress-induced activation of GATA-4 binding in adult heart. J Biol Chem 279: 24852-24860, 2004.

29. Condorelli G, Drusco A, Stassi G, et al: Akt induces enhanced myocardial contractility and cell size in vivo in transgenic mice. Proc Natl Acad Sci USA 99: 12333-12338, 2002.

30. Morisco C, Seta K, Hardt SE, Lee Y, Vatner SF and Sadoshima J: Glycogen synthase kinase 3beta regulates GATA4 in cardiac myocytes. J Biol Chem 276: 28586-28597, 2001.

31. Smeets PJ, Teunissen BE, Willemsen PH, et al: Cardiac hypertrophy is enhanced in PPAR alpha-/- mice in response to chronic pressure overload. Cardiovasc Res 78: 79-89, 2008.

32. Miyamoto T, Takeishi Y, Takahashi H, et al: Activation of distinct signal transduction pathways in hypertrophied hearts by pressure and volume overload. Basic Res Cardiol 99: 328-337, 2004.

33. Yang D, Ma S, Tan Y, et al: Adrenergic receptor blockade-induced regression of pressure-overload cardiac hypertrophy is associated with inhibition of the calcineurin/NFAT3/GATA4 pathway. Mol Med Rep 3: 497-501, 2010. 